\title{
Upregulation of MEK5 by Stat3 promotes breast cancer cell invasion and metastasis
}

\author{
FANG LIU ${ }^{1,2}, \mathrm{HAO}_{\mathrm{ZHANG}}^{3}$ and $\mathrm{HUI} \mathrm{SONG}^{1}$ \\ ${ }^{1}$ Xi'an Jiaotong University Suzhou Academy, Suzhou, Jiangsu 215123, P.R. China; \\ ${ }^{2}$ The University of Texas MD Anderson Cancer Center, Houston, TX 77030, USA; ${ }^{3}$ Shantou University \\ Medical College Cancer Research Center, Shantou, Guangdong 515041, P.R. China
}

Received May 13, 2016; Accepted October 3, 2016

DOI: $10.3892 /$ or.2016.5256

\begin{abstract}
Mitogen extracellular-signal-regulated kinase kinase 5 (MEK5) plays an important role in promoting cell proliferation and tumorigenesis. The aberrant expression of MEK5 has been reported in various malignant diseases including cancers of breast, prostate, lung, colorectal and brain. However, the function and regulation of MEK5 signaling pathway are ambiguous and remain elusive with respect to its oncogenic roles in various cancers, especially in the regulation of the initiation and progression of cancer invasion and metastasis. Ectopic expression of MEK5 or knockdown of MEK5 by shRNA with in vitro cell based models demonstrated the role of MEK 5 in regulation of epithelial mesenchymal transition (EMT) and breast cancer invasion and metastasis. Here, we show that MEK5 upregulated by Stat3 promotes breast cancer cell invasion through EMT. Further study demonstrated that Stat 3 could bind to promoter region of MEK5 and enhanced MEK5 transcription and expression. In addition, the phosphorylation of MEK5 significantly increased in breast cancer cells corresponding to metastatic capability of breast cancer cells. The depletion of MEK 5 by shRNA significantly decreased breast cancer invasion. Ectopic expression of MEK5 could confer non-invasive breast cancer cells to become invasion capable cells. Moreover, the phosphorylation of Erk5, a MEK5-regulated downstream kinase, was also upregulated consistent with the increased level of active MEK5. Our studies provide insights into a molecular mechanism by which MEK 5 transcriptionally upregulated by Stat 3 augments breast cancer cell EMT, which subsequently enhances cancer cell invasion and metastasis. This finding may suggest that Stat 3 and MEK5/Erk5 pathways could be an effective therapeutic target for inhibition of breast cancer invasion and metastasis.
\end{abstract}

Correspondence to: Dr Hui Song, Xi'an Jiaotong University Suzhou Academy, 99 Ren'ai Road, D101, Suzhou Industry Park, Suzhou, Jiangsu 215123, P.R. China

E-mail: sunny_um@yahoo.com

Key words: breast cancer, MEK5, Stat3, invasion, epithelial mesenchymal transition, metastasis

\section{Introduction}

The mitogen extracellular-signal-regulated kinase kinase 5/extracellular-signal-regulated kinase 5 (MEK5/Erk5) signal pathway was first characterized in 1995 (1) and categorized as a member of MEK/Erk family. MEK5 is a 444 amino acid protein with a mass of $50 \mathrm{kDa}(\mathrm{MEK} 5 \alpha)$ and $40 \mathrm{kDa}$ (MEK5 $\beta$ ), respectively (2). MEK5 protein conserves a partial domain of other MEK family kinases such as MEK1 and MEK2 with an overall 40\% homology (1). Physiologically, MEK5/Erk5 pathway has its functional role during normal muscle and neuronal development (3-5). It has been reported that MEK5 regulates skeletal and myocardium development through upregulation of Erk5 and MEF2 (6). Recent studies showed that targeted deletion of MEK5 led to embryonic lethality due to blockade of the MEK5/Erk5 signal pathway, which normal cardiac and embryonic development depends on $(7,8)$. These studies suggest that MEK5/Erk5 is an essential regulatory signal pathway for stem cell survival. MEK5 is the only known and characterized Erk5 kinase direct regulator. MEK5/Erk5 signaling pathway regulates a number of transcription factors. For example, MEF2, c-Fos, Fra-1 and NF- $\kappa$ B (6,9-11). Muscle differentiation and neuronal survival have been implicated to be associated with Erk5-regulated MEF2 signaling in vitro studies (7).

In addition, a few reports demonstrated that MEK5 protein was overexpressed and associated with certain types of cancer $(12,13)$, for example, cancers of breast, lung, colorectal, prostate and leukemia and neuroblastoma (14-22). Besides, MEK5/Erk5 signal pathway has been reported to regulate angiogenesis and tumor cell proliferation $(12,23)$. Notably, MEK5 has also been observed to play a role in EMT during breast cancer progression and metastasis by proteomics analysis $(16,24,25)$. However, the function and regulation of MEK5 signaling in cancer metastasis remain to be elucidated in detail.

With respect to the regulation of MEK5 upstream and downstream signal pathways, some reports demonstrated that MEK5 was regulated by TNF- $\alpha / J N K$ signal pathway $(26,27)$. MEKK2 and MEKK3 have been demonstrated as MEK5/Erk5 direct upstream regulators (28-30). It has been proved that MEK5 signaling function acts through regulating MEF2C, a member of the MEF2 transcription factor family (6). MEK5 
has been reported to promote prostate cancer metastasis through upregulation of MMP9 (14). Erk5 is MEK5-regulated immediate downstream kinase that was responsible for further activating MMP-9, which functions to enhance cancer cell migration and invasion. Moreover, MEK5 expression was also associated with poor prognosis in prostate cancer patients. MEK5 is also overexpressed in breast cancer (16), especially in cells that Stat3 signaling is persistently activated (31). The overexpression of constitutively active Stat3 (Stat3C) could upregulate total level of MEK5 protein expression and activation in breast cancer, suggesting that Stat 3 might participate in MEK5 upstream regulation in development of breast cancer. Stat 3 is frequently expressed and activated in triple-negative breast cancer (TNBC). In addition, MEK5 direct downstream target, Erk5, has been identified as an important factor responsible for poor prognosis and low survival rate of TNBC. This evidence suggests that MEK5/Erk5 inhibitors could have potential to mitigate malignancy and improve outcomes of TNBC (32-34). Therefore, therapeutical targets related to kinases, which are responsible for EMT such as Erk5 and MAPK7, are attractive for developing new generation of anticancer drugs $(34,35)$.

In order to understand cellular regulatory role of MEK5 signaling in breast cancer invasion and metastasis, we compared MEK5 expression and activation in comparison of invasive and non-invasive breast cancer in the present study. Our data demonstrated that MEK5 was activated in invasive breast cancer cell lines that we tested, but not non-invasive breast cancer cell line and/or immortalized cell line. Ectopic expression of MEK5 could lead to non-invasive MCF7 breast cancer cells to change morphology through EMT. The knockdown of MEK5 in highly invasive MDA-MB-231 cells resulted in the loss of its invasive and metastatic ability. The mechanism study suggested that active Stat 3 was closely correlated with high level of MEK5 expression and activation. Together, our observations suggested that MEK5 expression was transcriptionally upregulated by Stat 3 activation.

Although active Stat 3 was critical to activate MEK5 and further induce cancer cell EMT, Stat 3 activation was not essential to initiate these cellular processes. Instead, we provided evidence to show that the activation of MEK5 through serine/threonine phosphorylation was essential for the initiation of cell invasion and metastasis. Taken together, the present study unveiled a new insight into the mechanism by which transcriptionally upregulated MEK 5 by active Stat 3 was essential to initiate cell EMT and resulted in cancer cell invasion and metastasis. The significance of our observations may implicate that the blockade of Stat 3 and MEK5/Erk5 pathways could potentially benefit the prevention of breast cancer metastasis $(13,24,25,36)$.

\section{Materials and methods}

Cell lines and chemicals. MCF-7, MCF10A, MDA-MB-231 and MDA-MB-468 cell lines were purchased from the American Type Culture Collection (ATCC; http://www.atcc. org/). Spontaneous immortalized MCF10A breast cells (37) were cultured as previously described (31). Breast carcinoma cell lines, MCF-7, MDA-MB-231 and MDA-MB-468, were cultured in Dulbecco's modified Eagle's medium (DMEM) containing $10 \%$ fetal bovine serum (FBS) with appropriate antibiotics. All chemical reagents were purchased from SigmaAldrich (St. Louis, MO, USA) or Thermo Fisher Scientific (Pittsburgh, PA, USA) and were of analytically pure grade if not otherwise noted.

Western blot analysis. For western blot analysis, $100 \mu \mathrm{g}$ of cell lysate was resolved on SDS-PAGE and transferred to PVDF membrane. Antibodies against MEK5 (BD Transduction Laboratories, San Diego, CA, USA), phos-Stat3 (Tyrosine705), Stat3, Erk5, phospho-Erk5 (Cell Signaling Technology, Inc., Beverly, MA, USA), phospho-MEK5 (S311/T315), phosphoMEKK1, MEKK1, MEKK2, MEKK3 (Abcam, Cambridge, MA, USA), phospho-MEKK2 (Thermo Fisher Scientific), phospho-MEKK3 (Sigma-Aldrich), E-cadherin, N-cadherin, Snail, Slug, vimentin (Santa Cruz Biotechnology, Santa Cruz, CA, USA) and tubulin (Sigma-Aldrich) were used to detect corresponding proteins, respectively.

Cell invasion assay. Cell invasion assay was performed using an invasion chamber system (BD Transduction Laboratories) following the manufacturer's instructions. In brief, $2.5 \times 10^{4}$ cancer cells in $0.5 \mathrm{ml}$ culture medium were seeded into upper compartment in two layer chamber invasion plates. The cells were cultured for $24 \mathrm{~h}$ prior to staining of the Matrigel membrane. Cell penetration through the membrane was detected by staining the cells on the porous membrane with a Diff-Quik stain kit (Dade Behring, Inc., Newark, DE, USA). Each experimental group was performed at least in triplicate. The results are denoted as mean \pm standard deviation (SD).

Chromatin immunoprecipitation (ChIP) assays and real-time $P C R$. To detect Stat3 binding with MEK5 promoter region, a ChIP assay kit (Upstate Biotechnology, Lake Placid, NY, USA) was employed in the present study. The assay was performed according to the manufacturer's instructions and protocols previously described (31). For measuring MEK5 expression, a real-time PCR was performed and relative expression level was calculated accordingly.

Infection of breast cancer cells by viral constructs. The viral plasmid constructs, pWZL-MEK5 and pMX-Stat3C were used for expression of MEK5 and active Stat3C, respectively. The construct for expression of constitutively activated MEK5DD (S311D/T315D) was generated by site-directed mutagenesis and confirmed by DNA sequencing. MEK5 knockdown was achieved by using lentiviral shRNA particles (Santa Cruz Biotechnology) following the manufacturer's instructions. Breast cancer MCF-7 or MD-MB-231 cells were infected for expression of Stat 3 or MEK5 following standard procedures. The infected cells were selected by neomycin for 3 weeks and pooled for next experiments or further infection by lentiviral MEK5 shRNA, if needed. The MDA-MB-231 cells were infected by lentiviral MEK5 shRNA and selected by puromycin for two weeks and pooled for assays.

Immunohistochemical (IHC) analysis. Human breast cancer tissue array slides were obtained from the National Cancer Institute (Philadelphia, PA, USA) and Chemicon International, Inc. (Temecula, CA, USA). Immunostaining was conducted 


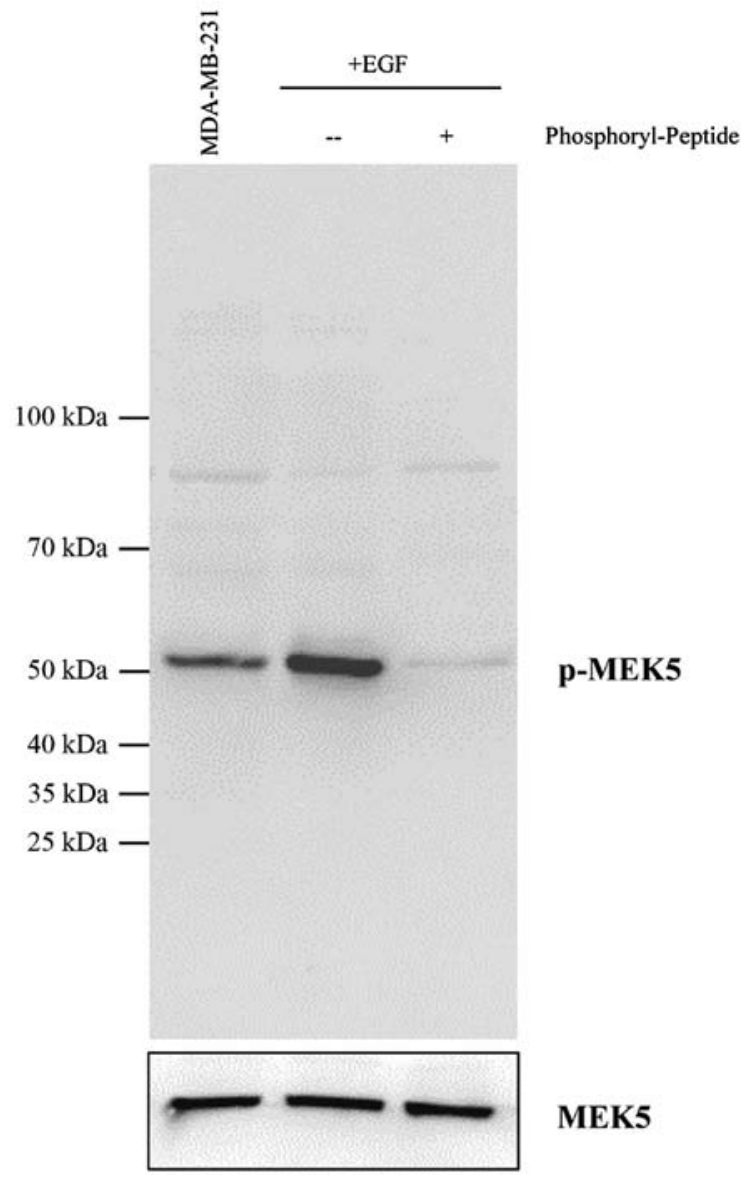

Figure 1. Validation of phospho-MEK5 antibody. MDA-MB-231 cells were treated with EGF ( $25 \mathrm{ng} / \mathrm{ml}$ for $20 \mathrm{~min}$ ) and then lysated for western blotting. Western blotting was performed by using phospho-MEK5 in the presence or the absence of the peptide that was used for immunizing animals for antibody production.

by using Chemicon IHC Select ${ }^{\mathrm{TM}}$ detection system (Chemicon International). The array slides were stained and probed by using relevant antibodies according to the standard procedures and the manufacturer's instructions.

Statistical analysis. The data are described as mean \pm standard derivation or odds ratio. Statistical analyses were performed by the Student's t-test, ANOVA and Chi-square analysis. $\mathrm{P}<0.05$ was considered as statistically significant.

\section{Results}

MEK5 is overexpressed in Stat 3 activated breast cancer cells, but not in normal breast epithelial cells or non-invasive cells. We previously showed that MEK5 was overexpressed in a majority of breast cancer cells that we tested (31). To prove that this is the case in breast cancer tissues, we first evaluated an appropriate anti-phospho-MEK5 antibody that is suitable for tissue immunostaining (Fig. 1). A group of samples with 106 cases, including 10 normal breast tissues, 36 triple-positive tissues and 60 TNBCs, were studied in this investigation. The results showed that odds ratio of Stat 3 and MEK5 phosphorylation in breast cancer tissues is $36.47(\mathrm{P}<0.001)$ and 25.92 $(\mathrm{P}<0.001)$ respectively, which was significantly higher than that in normal tissues (Fig. 2). As Fig. 3A demonstrates, breast
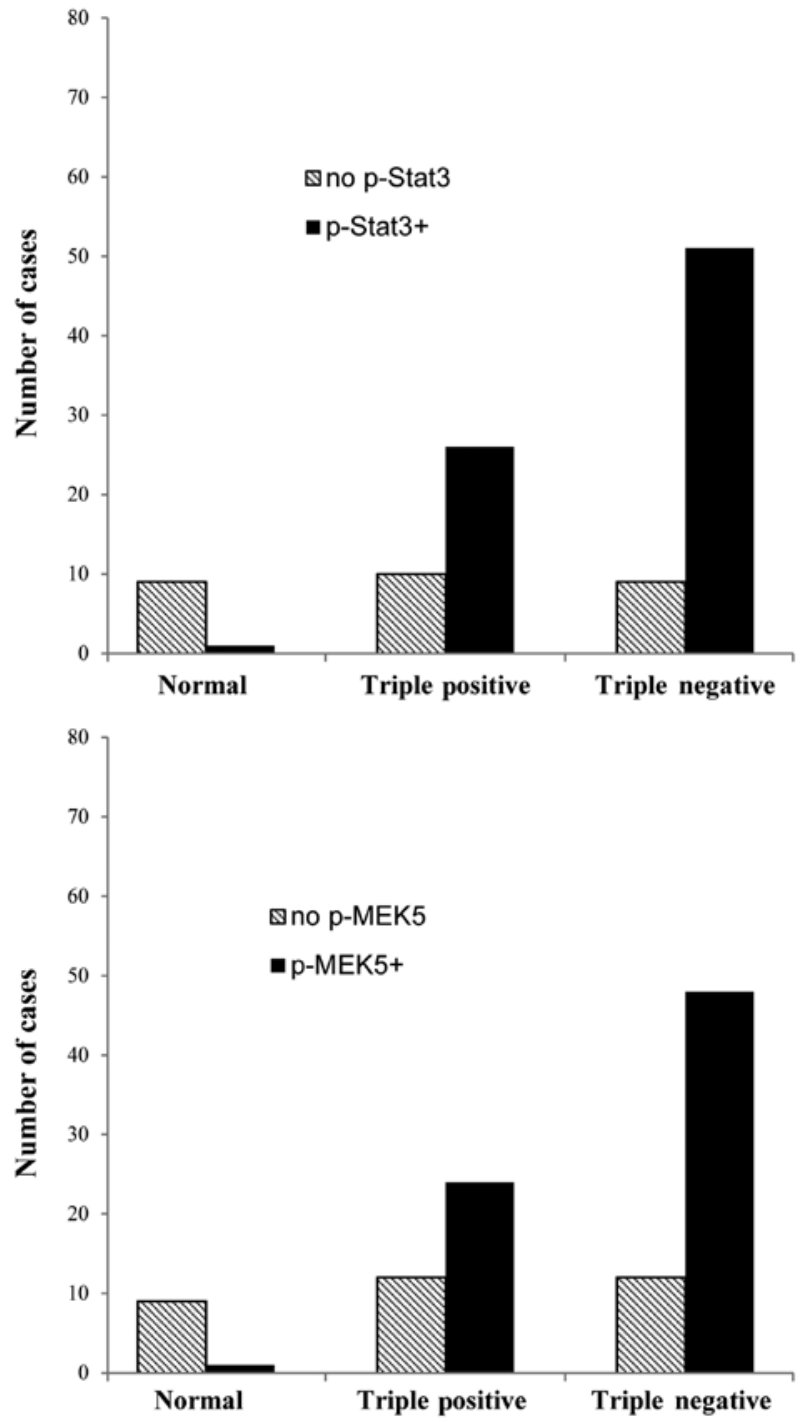

Figure 2. Breast tissues for immunostaining. A cohort of samples with 10 normal breast tissues, 36 breast cancer tissues with triple-positive staining $\left(\mathrm{ER}^{+}, \mathrm{PR}^{+}\right.$and $\left.\mathrm{HER}^{+}\right)$and $60 \mathrm{TNBCs}$ were performed in this investigation. Odds ratio and Chi-square analysis were performed for analyzing the significance of difference of phosphorylated-Stat 3 and phosphorylated-MEK5 in breast cancer tissues.

tissue IHC staining showed that active Stat 3 was correlated with high level of MEK5 expression and activation. MEK5 is overexpressed and activated in invasive breast cancer cell lines MDA-MB-231 and MDA-MB-468, but not in immortalized MCF10A cells. Although MEK5 protein could be detected in non-invasive MC-F7 cells (Fig. 3B), the phosphorylation of MEK5 was undetectable, suggesting that MEK5 might be inactive. Further studies identified that constitutively activated Stat 3 could increase phosphorylation of MEKK2/3 but not MEKK1. MEK5/Erk5 specific inhibitor, BIX02189 can reduce Stat3-mediated phosphorylation of MEK5/Erk5. However, phosphorylation of MEKK2/3 was not affected by such an inhibitor (Fig. 3C). Ectopic expression of either active Stat3C, which could mimic active phosphorylated Stat 3 by constitutively forming dimers or MEK5 caused the change of cell morphology. The inhibition of MEK5 by MEK5/Erk5 inhibitor could block such a change of cell morphology (Fig. 3D). Together, suggesting that both active Stat 3 and MEK5 are able 
A
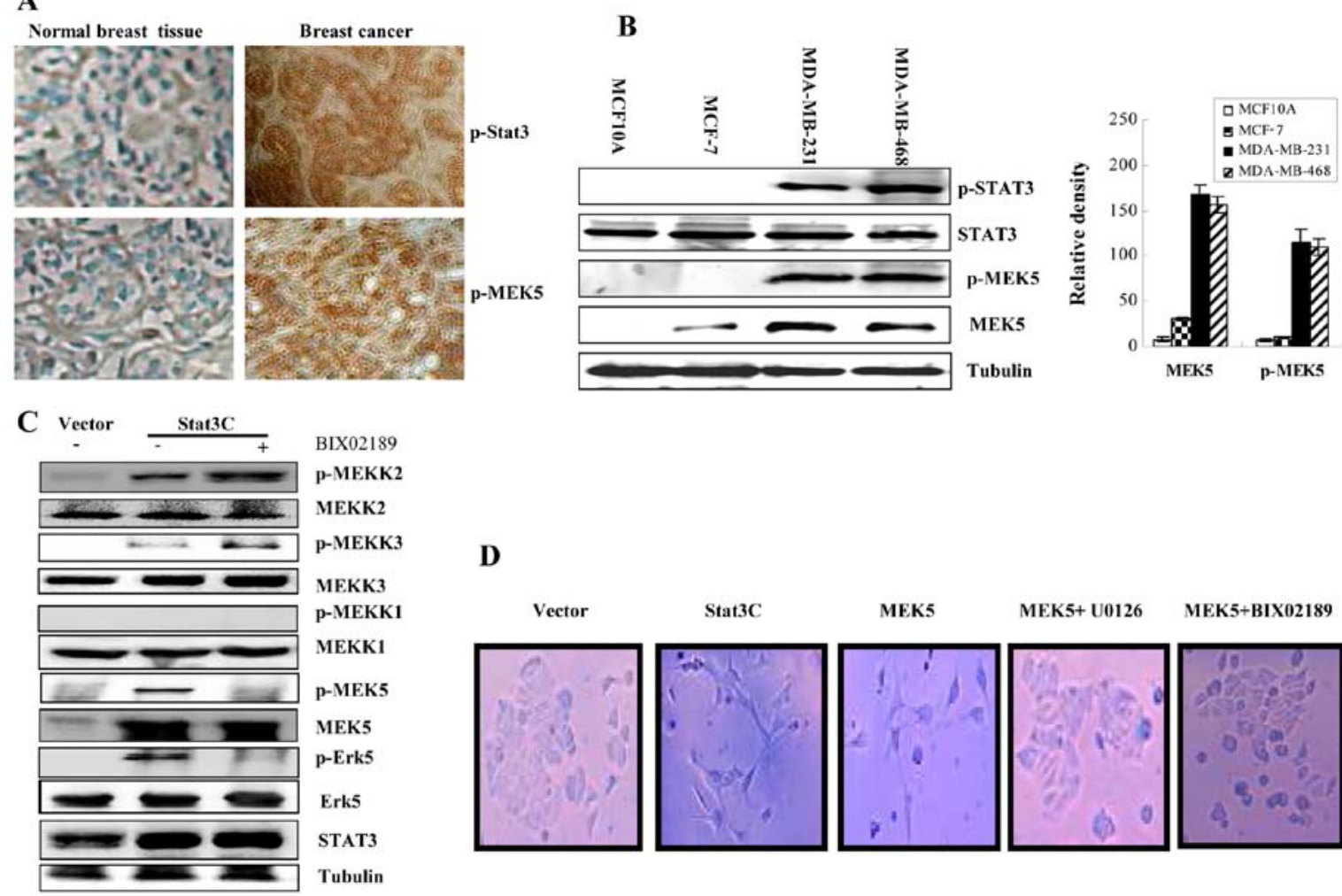

D

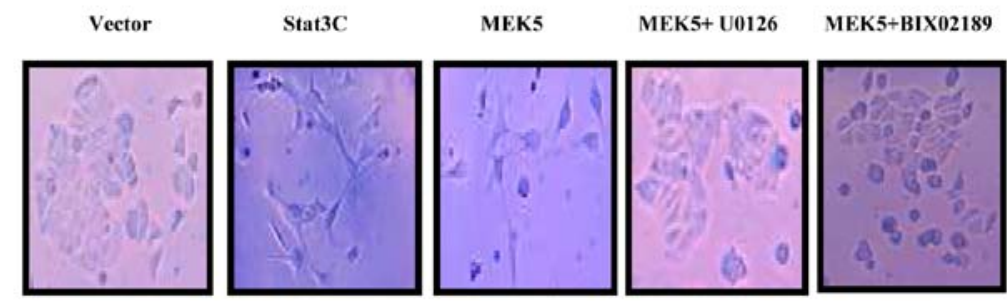

Figure 3. MEK5 expression and activation are correlated with activated Stat 3 in breast cancer cells. (A) Tissue IHC using antibodies as indicated in the figure. For detailed information see the Materials and methods. (B) Western blotting by using cell lysates as indicated. (C) Western blotting by using cell lysates from MCF-7 expressing Stat3C. (D) MCF-7 cell morphology changes in the cells that express active Stat3C or MEK5 with or without MEK5/Erk5 inhibitors U0126 and BIX02189.

to induce cancer cells to change morphology and MEK5 may be activated by MEKK2/3 and play an important role in this cellular process.

MEK5 promotes non-invasive MCF-7 cells to become invasive cancer cells. MCF-7 cells are non-invasive breast cells and were negative for EMT markers such as vimentin (38-40). However, upon ectopic expression either Stat3C or MEK5, MCF-7 cells became invasive (Fig. 4A). Western blot analysis from cell lysates showed that ectopic expression of both Stat3C and MEK5 could activate the MEK5/Erk5 signal pathway through serine or threonine phosphorylation (Fig. 4B). Both knockdown of MEK 5 by shRNA and inhibition of MEK5 by a MEK5/Erk5 inhibitor BIX02189 could block Stat3Cinduced invasiveness in the cells that ectopically expressed active Stat3C (Fig. 4C and D), suggesting that active Stat3 acted through activation of MEK5 and MEK5/Erk5 signal pathway played a key role in this cellular process. Together, these observations indicate that MEK5 may be essential for regulating State 3 mediated breast cancer cell invasion and metastasis.

Knockdown of MEK5 inhibits cell invasion of invasive MDA-MB-231 breast cancer cells. MDA-MB-231 cells express high level of activated Stat 3 and MEK5 and aggressively metastasize to the lung. Knockdown of MEK5 or treatment by a MEK5 inhibitor could significantly inhibit its invasive phenotype through blockade of activation of MEK5/ Erk5 pathway (Fig. 5A and B). The inhibition of Stat3C by a
Stat3 inhibitor, STA-21 (41), also reduced its invasive ability (Fig. 5C and D). However, in the cells that expressed constitutively active MEK5DD (S311D/T315D), STA-21 was not able to inhibit cell invasion. This result suggests that Stat 3 functioned through MEK5 activation, which was essential for EMT and cell invasion. However, the inhibition of MEK 5 by both shRNA and a MEK5/Erk5 inhibitor, BIX02189, significantly reduced invasion (Figs. 4 and 5). Even though MDA-MB-231 expressed constitutively activated Stat $3 \mathrm{C}$, the blockade of MEK5 was still able to impede cell invasion (Fig. 4C). These results suggest that MEK5 plays a key role in the regulation of breast cancer cell invasion. To summarize, this evidence indicates that Stat3-induced cancer cell invasion and metastasis may, at least in part, depend on activation status of MEK5. In other words, MEK5 was essential for enabling breast cancer invasion and further metastasis.

Stat 3 transcriptionally upregulates MEK5 expression. We previously reported that Stat3 directly upregulated MEK5 expression through microarray analysis. As reported by us (31), there are twelve candidate Stat3-binding sites that exist in the $3.2 \mathrm{~kb}$ MEK5 promoter region. Ten out of the twelve Stat3-binding sites are located at the position from -1776 to -1037 upstream of the transcription initiation site. The sequence TTCTGGAAA between -1770 and -1762 was used for ChIP assay. As showed in Fig. 6A, interferons and active Stat3C were able to induce MEK5 expression suggesting that Stat3 directly regulated MEK5 in its promoter region (Fig. 6A). Real-time PCR also demonstrated that Stat3C 
$\mathbf{A}$
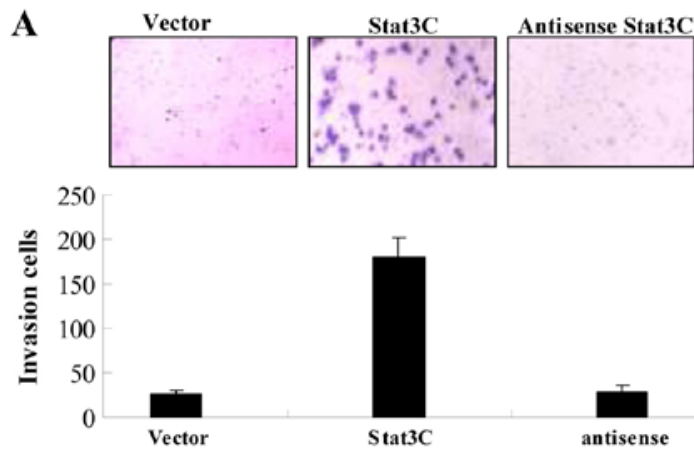

C
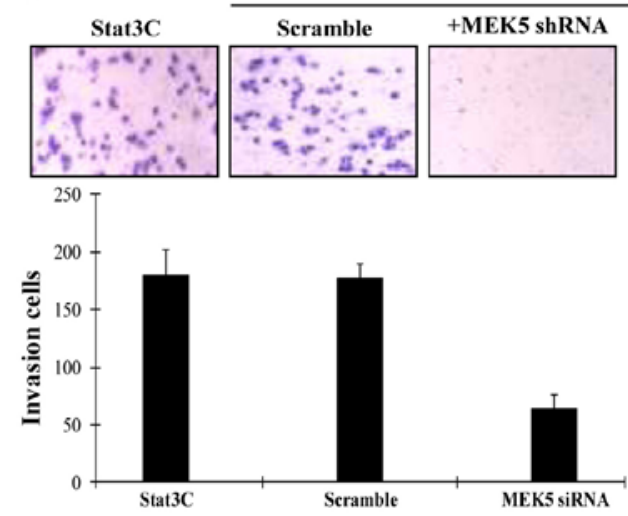
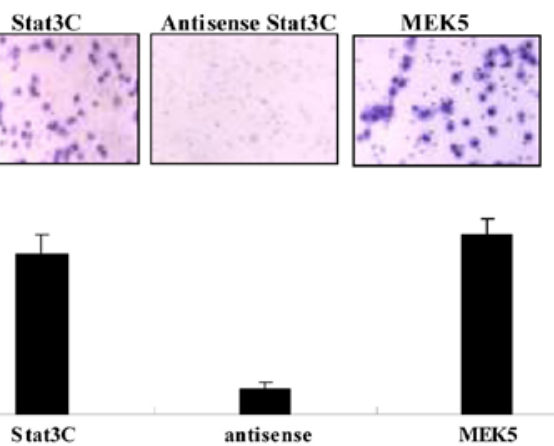

antisense

MEK5

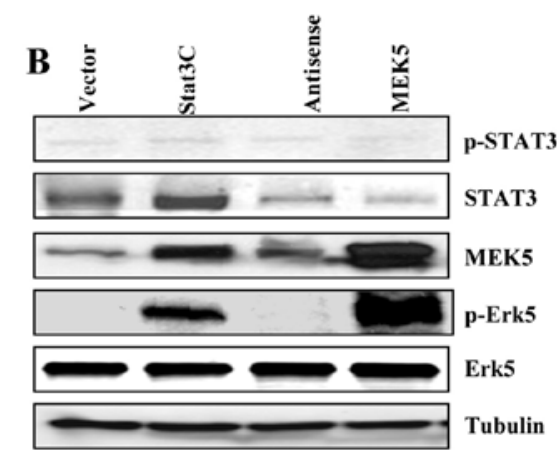

D
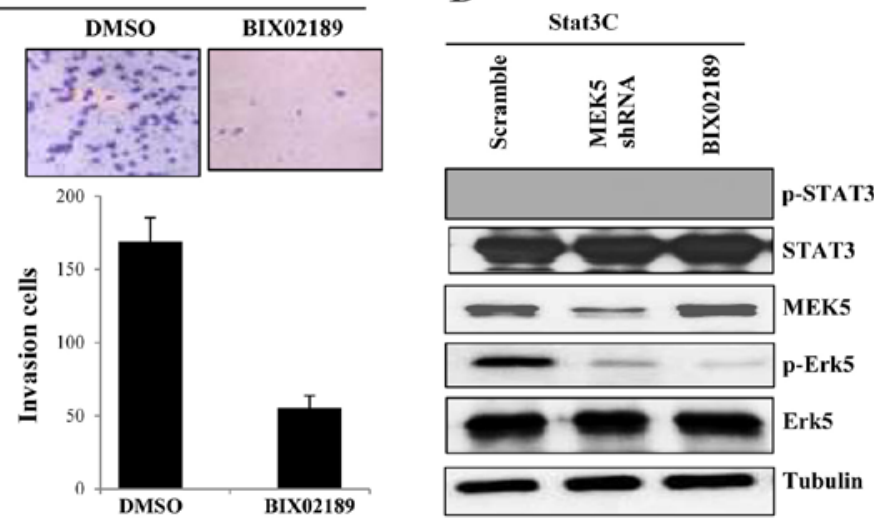

Figure 4. Stat 3 regulates breast cancer cell invasion through upregulation and activation of MEK5. (A) MCF-7 cells with expression of either active Stat3C or MEK5 or antisense Stat3C were used for invasion assay. (B) Western blotting using cell lysates from (A). (C) MCF-7 cells with expression of constitutively activated Stat3C were treated with either knockdown of MEK 5 by shRNA or $5 \mu \mathrm{M}$ BIX02189 for $24 \mathrm{~h}$. Then, invasion assay was performed by using the cells described above. (D) Western blotting using cell lysates from C. For invasion assay, each group was performed at least in triplicate. The results are described as mean $\pm \mathrm{SD}$.

$\mathbf{A}$
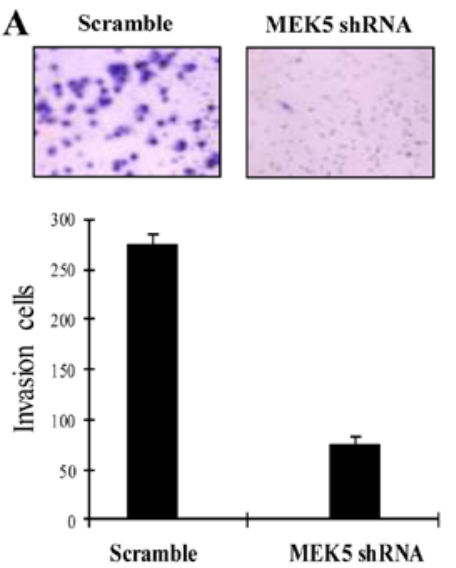

C

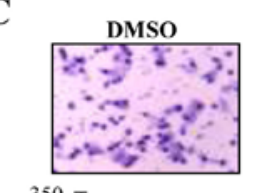

STA-21
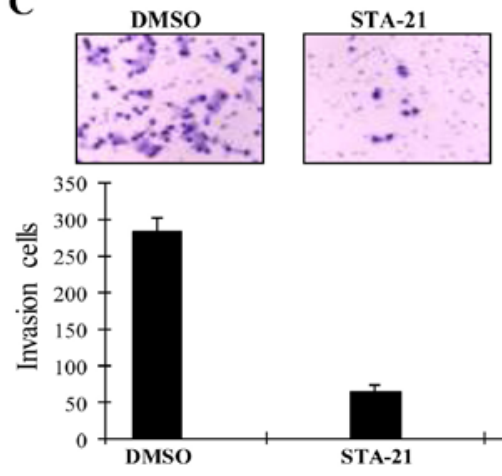
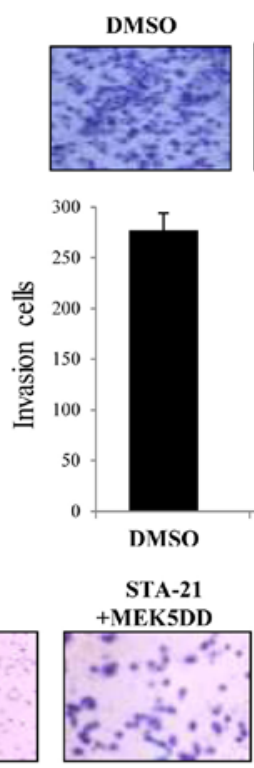

STA-21

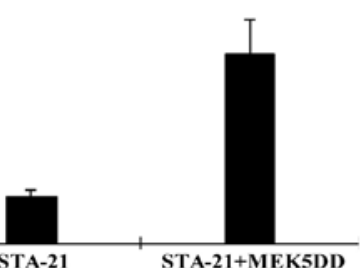

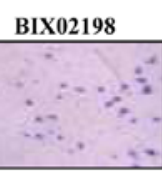

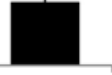

BIX02189

D

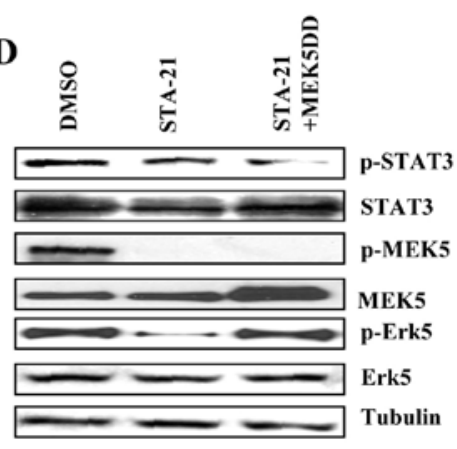

Figure 5. MEK5 plays an essential role in controlling cancer cell invasion in vitro. (A) MDA-MB-231 cells were treated by either knockdown of MEK5 or $5 \mu \mathrm{M}$ BIX02189 and then subjected to invasion assay. (B) Western blotting using cell lysates from (A). (C) MDA-MB-231 cells with expression of activated MEK5 (MEK5DD) were treated with $20 \mu \mathrm{M} \mathrm{STA}-21$ (a Stat3 inhibitor) and then subjected to invasion assay. (D) Western blotting using cell lysates from (C). 
A

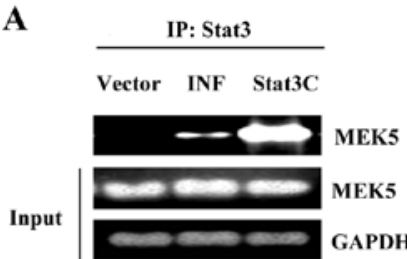

C

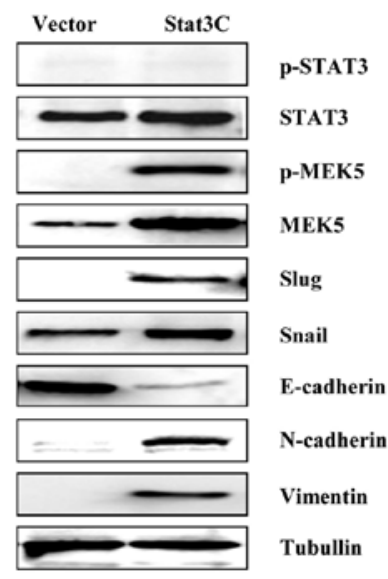

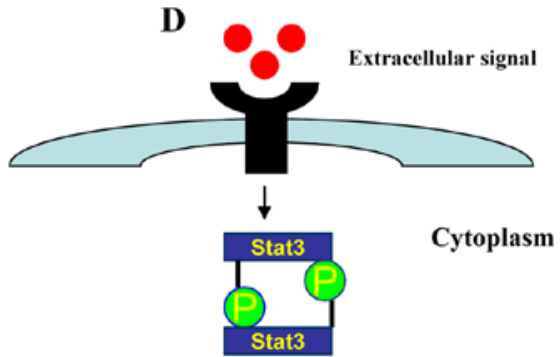

B

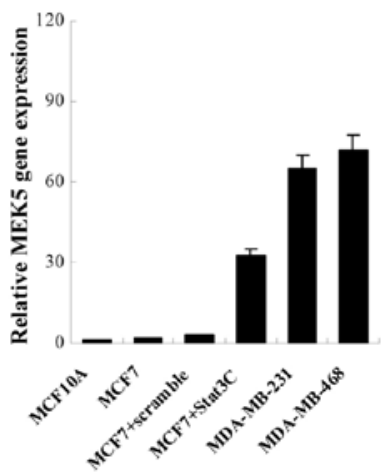

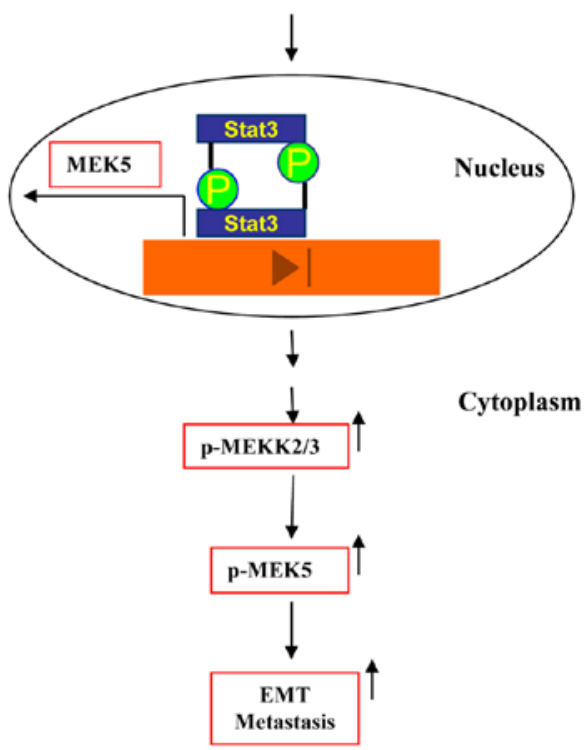

Figure 6. Transcriptional upregulation of MEK 5 by Stat3 results in enhancement of tumor cell EMT and metastasis. (A) ChIP assay was performed as previously described (32). (B) Real-time PCR was performed to determine MEK5 mRNA expression in different cell lines. (C) Western blotting was performed by using antibodies as indicated. (D) Proposed model for Stat3-MEK5-regulated breast cancer cell EMT and metastasis.

enhanced MEK5 mRNA expression (Fig. 6B). Further study showed that active Stat $3 \mathrm{C}$ was a causative factor that increased MCF-7 cells to express EMT markers, including increased expression of MEK5, p-MEK5, N-cadherin, vimentin, slug, snail and a decrease of E-cadherin (Fig. 6C). Together, these data support that active Stat3-mediated upregulation of MEK5 directly contributed to cancer cell EMT, which subsequently resulted in cancer invasion and metastasis. To illustrate this concept, we propose a model that explains a possible mechanism of MEK5-induced breast cancer cell EMT and metastasis (Fig. 6D). In summary, either stimulus-activated or constitutively activated Stat 3 could transcriptionally upregulate MEK5 expression and activate MEK5 through increasing phosphorylation of MEKK2/3. Subsequently, an increased level of active MEK5 causes breast cancer cell EMT and metastasis. During this process, MEK5/Erk5 plays an essential role in breast cancer invasion and metastasis.

\section{Discussion}

Stat 3 plays a critical role in development of normal embryonic stem cells and tumorigenesis. Constitutively activated Stat 3 was linked to various types of cancer such as cancers of the breast, prostate, lung, brain, colorectal and leukemia. Moreover, Stat 3 has also been reported to be involved in the initiation and progression of cancer stem cells, including glioblastoma, hepatoma, sarcoma, breast, head and neck and skin cancer (42-47). In addition, Stat3 seems to be a key modulator that regulates cancer cell EMT and promotes cancer metastasis $(16,46,48)$. However, our observations suggest that Stat3 has to act through MEK5 in order to trigger breast cancer invasion and metastasis. It is of interest that similar observation of MEK5-promoted EMT has also been reported $(16,24)$. Therefore, this evidence indicates that Stat3-MEK5/Erk5 pathway has a critical role in the regulation of EMT, thereafter cancer metastasis.

Although MEK5/Erk5 pathway plays an important role in various cellular processes $(1,2,49,50)$, little is known about how MEK5 functions during the development of tumorigenesis and/or cancer metastasis. MEK5 overexpression has been reported in invasive breast and prostate cancer $(14,32)$. Especially in prostate cancer, MEK5 has been reported to play a pivotal role in prostate cancer progression and metastasis. However, the underlying molecular mechanism by which MEK5 regulates these cellular processes remains elusive. The understanding of the role of MEK5/Erk5 pathway in cancer metastasis may help to develop an effective cancer therapeutic regimen and prevent cancer from reoccurrence and/or metastasis.

In the present study, we observed that constitutively activated Stat 3 not only increased the level of MEK5 expression, but also enhanced MEK5 phosphorylation by increasing phosphorylated MEKK2 and MEKK3. One possibility could be that Stat3-mediated stress responses are similar to oxidative stress response. Further study needs to be conducted in order to understand molecular mechanisms of how activated Stat3 can augment phosphorylation of MEKK2/3. 
MEK5 played an essential role in controlling cancer cell EMT and subsequent metastasis. The evidence from ectopic expression and knockdown of MEK5 suggested that MEK5 is a key player in these processes. Although Stat 3 constitutive activation alone could activate the cancer cell EMT process, blockade of MEK 5 by either shRNA or a small molecule inhibitor could impede this development, suggesting that Stat 3 acted through MEKK2/3-activated MEK5 (Figs. 3C and 6D). Taken together, these data suggest that MEK5 may play a pivotal role in the initiation of cancer cell invasion and metastasis. MEK5 participated in regulation of breast cancer progression and metastasis through increasing cancer cell EMT. Stat3 along with MEK5 could possibly be effective therapeutic targets for the treatment of breast cancer, especially TNBCs. The implications of the present study may suggest that blockade of Stat 3 and MEK5/Erk5 pathways along with other conventional interventions could potentially benefit breast cancer therapy and improve therapeutic outcomes by blocking cancer cell metastasis.

\section{Acknowledgements}

The present study was supported by the National Natural Science Foundation of China NSFC 31271495 (to H.S.).

\section{References}

1. Zhou G, Bao ZQ and Dixon JE: Components of a new human protein kinase signal transduction pathway. J Biol Chem 270: 12665-12669, 1995

2. English JM, Vanderbilt CA, Xu S, Marcus S and Cobb MH: Isolation of MEK5 and differential expression of alternatively spliced forms. J Biol Chem 270: 28897-28902, 1995.

3. Dinev D, Jordan BW, Neufeld B, Lee JD, Lindemann D, Rapp UR and Ludwig S: Extracellular signal regulated kinase 5 (ERK5) is required for the differentiation of muscle cells. EMBO Rep 2: 829-834, 2001

4. Shalizi A, Lehtinen M, Gaudilliere B, Donovan N, Han J, Konishi Y and Bonni A: Characterization of a neurotrophin signaling mechanism that mediates neuron survival in a temporally specific pattern. J Neurosci 23: 7326-7336, 2003.

5. Liu L, Cavanaugh JE, Wang Y, Sakagami H, Mao Z and Xia Z: ERK5 activation of MEF2-mediated gene expression plays a critical role in BDNF-promoted survival of developing but not mature cortical neurons. Proc Natl Acad Sci USA 100: 8532-8537, 2003.

6. Kato Y, Kravchenko VV, Tapping RI, Han J, Ulevitch RJ and Lee JD: BMK1/ERK5 regulates serum-induced early gene expression through transcription factor MEF2C. EMBO J 16: 7054-7066, 1997.

7. Wang X, Merritt AJ, Seyfried J, Guo C, Papadakis ES, Finegan KG, Kayahara M, Dixon J, Boot-Handford RP, Cartwright EJ, et al: Targeted deletion of mek 5 causes early embryonic death and defects in the extracellular signal-regulated kinase 5/myocyte enhancer factor 2 cell survival pathway. Mol Cell Biol 25. 336-345, 2005.

8. Yan L, Carr J, Ashby PR, Murry-Tait V, Thompson C and Arthur JS: Knockout of ERK5 causes multiple defects in placental and embryonic development. BMC Dev Biol 3: 11-32, 2003.

9. Terasawa K, Okazaki K and Nishida E: Regulation of c-Fos and Fra-1 by the MEK5-ERK5 pathway. Genes Cells 8: 263-273, 2003.

10. Pearson G, English JM, White MA and Cobb MH: ERK5 and ERK2 cooperate to regulate NF-kappaB and cell transformation. J Biol Chem 276: 7927-7931, 2001

11. Kato Y, Zhao M, Morikawa A, Sugiyama T, Chakravortty D, Koide N, Yoshida T, Tapping RI, Yang Y, Yokochi T, et al: Big mitogen-activated kinase regulates multiple members of the MEF2 protein family. J Biol Chem 275: 18534-18540, 2000.

12. Lochhead PA, Gilley R and Cook SJ: ERK 5 and its role in tumour development. Biochem Soc Trans 40: 251-256, 2012.
13. Drew BA, Burow ME and Beckman BS: MEK5/ERK5 pathway: The first fifteen years. Biochim Biophys Acta 1825: 37-48, 2012.

14. Mehta PB, Jenkins BL, McCarthy L, Thilak L, Robson CN, Neal DE and Leung HY: MEK5 overexpression is associated with metastatic prostate cancer, and stimulates proliferation, MMP-9 expression and invasion. Oncogene 22: 1381-1389, 2003.

15. Ramsay AK, McCracken SR, Soofi M, Fleming J, Yu AX, Ahmad I, Morland R, Machesky L, Nixon C, Edwards DR, et al: ERK5 signalling in prostate cancer promotes an invasive phenotype. Br J Cancer 104: 664-672, 2011.

16. Zhou C, Nitschke AM, Xiong W, Zhang Q, Tang Y, Bloch M, Elliott S, Zhu Y, Bazzone L, Yu D, et al: Proteomic analysis of tumor necrosis factor-alpha resistant human breast cancer cells reveals a MEK5/Erk5-mediated epithelial-mesenchymal transition phenotype. Breast Cancer Res 10: R105, 2008.

17. Qiu F, Yang L, Fang W, Li Y, Yang R, Yang X, Deng J, Huang B, Xie C, Zhou Y, et al: A functional polymorphism in the promoter of ERK5 gene interacts with tobacco smoking to increase the risk of lung cancer in Chinese populations. Mutagenesis 28: 561-567, 2013.

18. Diao D, Wang L, Wan J, Chen Z, Peng J, Liu H, Chen X, Wang W and Zou L: MEK5 overexpression is associated with the occurrence and development of colorectal cancer. BMC Cancer 16: $302,2016$.

19. Simões AE, Pereira DM, Gomes SE, Brito H, Carvalho T, French A, Castro RE, Steer CJ, Thibodeau SN, Rodrigues CM, et al: Aberrant MEK5/ERK5 signalling contributes to human

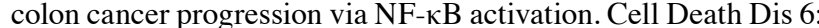
e1718, 2015

20. Mansour MA, Hyodo T, Ito S, Kurita K, Kokuryo T, Uehara K, Nagino M, Takahashi M, Hamaguchi $M$ and Senga T: SATB2 suppresses the progression of colorectal cancer cells via inactivation of MEK5/ERK5 signaling. FEBS J 282: 1394-1405, 2015.

21. Wang $X$, Pesakhov S, Harrison JS, Danilenko $M$ and Studzinski GP: ERK5 pathway regulates transcription factors important for monocytic differentiation of human myeloid leukemia cells. J Cell Physiol 229: 856-867, 2014.

22. Umapathy G, El Wakil A, Witek B, Chesler L, Danielson L, Deng X, Gray NS, Johansson M, Kvarnbrink S, Ruuth K, et al: The kinase ALK stimulates the kinase ERK5 to promote the expression of the oncogene MYCN in neuroblastoma. Sci Signal 7: ra102, 2014.

23. Giurisato E and Tournier C: Can tumor cells proliferate without ERK5? Cell Cycle 15: 619-620, 2016.

24. Antoon JW, Martin EC, Lai R, Salvo VA, Tang Y, Nitzchke AM, Elliott S, Nam SY, Xiong W, Rhodes LV, et al: MEK5/ERK5 signaling suppresses estrogen receptor expression and promotes hormone-independent tumorigenesis. PLoS One 8: e69291, 2013

25. Li J, Dong L, Wei D, Wang X, Zhang S and Li H: Fatty acid synthase mediates the epithelial-mesenchymal transition of breast cancer cells. Int J Biol Sci 10: 171-180, 2014.

26. Yoshizumi M, Abe J, Tsuchiya K, Berk BC and Tamaki T: Stress and vascular responses: atheroprotective effect of laminar fluid shear stress in endothelial cells: possible role of mitogenactivated protein kinases. J Pharmacol Sci 91: 172-176, 2003.

27. Dent P, Yacoub A, Fisher PB, Hagan MP and Grant S: MAPK pathways in radiation responses. Oncogene 22: 5885-5896, 2003.

28. Chayama K, Papst PJ, Garrington TP, Pratt JC, Ishizuka T, Webb S, Ganiatsas S, Zon LI, Sun W, Johnson GL, et al: Role of MEKK2-MEK5 in the regulation of TNF-alpha gene expression and MEKK2-MKK7 in the activation of c-Jun N-terminal kinase in mast cells. Proc Natl Acad Sci USA 98: 4599-4604, 2001.

29. Chao TH, Hayashi M, Tapping RI, Kato Y and Lee JD: MEKK3 directly regulates MEK5 activity as part of the big mitogenactivated protein kinase 1 (BMK1) signaling pathway. J Biol Chem 274: 36035-36038, 1999.

30. Sun W, Kesavan K, Schaefer BC, Garrington TP, Ware M, Johnson NL, Gelfand EW and Johnson GL: MEKK2 associates with the adapter protein Lad/RIBP and regulates the MEK5-BMK1/ERK5 pathway. J Biol Chem 276: 5093-5100, 2001.

31. Song $\mathrm{H}$, Jin $\mathrm{X}$ and Lin J: Stat 3 upregulates MEK5 expression in human breast cancer cells. Oncogene 23: 8301-8309, 2004.

32. Miranda M, Rozali E, Khanna KK and Al-Ejeh F: MEK5-ERK5 pathway associates with poor survival of breast cancer patients after systemic treatments. Oncoscience 2: 99-101, 2015.

33. Ortiz-Ruiz MJ, Álvarez-Fernández S, Parrott T, Zaknoen S, Burrows FJ, Ocaña A, Pandiella A and Esparís-Ogando A: Therapeutic potential of ERK5 targeting in triple negative breast cancer. Oncotarget 5: 11308-11318, 2014. 
34. Al-Ejeh F, Miranda M, Shi W, Simpson PT, Song S, Vargas AC Saunus JM, Smart CE, Mariasegaram M, Wiegmans AP, et al: Kinome profiling reveals breast cancer heterogeneity and identifies targeted therapeutic opportunities for triple negative breast cancer. Oncotarget 5: 3145-3158, 2014.

35. Javaid S, Zhang J, Smolen GA, Yu M, Wittner BS, Singh A, Arora KS, Madden MW, Desai R, Zubrowski MJ, et al: MAPK7 regulates EMT features and modulates the generation of CTCs. Mol Cancer Res 13: 934-943, 2015.

36. Li JQ, Xue H, Zhou L, Dong LH, Wei DP and Li H: Mechanism of fatty acid synthase in drug tolerance related to epithelialmesenchymal transition of breast cancer. Asian Pac J Cancer Prev 15: 7617-7623, 2014.

37. Song H, Ethier SP, Dziubinski ML and Lin J: Stat 3 modulates heat shock $27 \mathrm{kDa}$ protein expression in breast epithelial cells. Biochem Biophys Res Commun 314: 143-150, 2004.

38. Li QQ, Xu JD, Wang WJ, Cao XX, Chen Q, Tang F, Chen ZQ, Liu XP and Xu ZD: Twist1-mediated adriamycin-induced epithelial-mesenchymal transition relates to multidrug resistance and invasive potential in breast cancer cells. Clin Cancer Res 15: 2657-2665, 2009.

39. Kumar A, Xu J, Brady S, Gao H, Yu D, Reuben J and Mehta K: Tissue transglutaminase promotes drug resistance and invasion by inducing mesenchymal transition in mammary epithelial cells. PLoS One 5: e13390, 2010.

40. Cheng GZ, Chan J, Wang Q, Zhang W, Sun CD and Wang LH: Twist transcriptionally up-regulates AKT2 in breast cancer cells leading to increased migration, invasion, and resistance to paclitaxel. Cancer Res 67: 1979-1987, 2007.

41. Song H, Wang R, Wang S and Lin J: A low-molecular-weight compound discovered through virtual database screening inhibits Stat 3 function in breast cancer cells. Proc Natl Acad Sci USA 102: 4700-4705, 2005.

42. Villalva C, Martin-Lannerée S, Cortes U, Dkhissi F, Wager M, Le Corf A, Tourani JM, Dusanter-Fourt I, Turhan AG and Karayan-Tapon L: STAT3 is essential for the maintenance of neurosphere-initiating tumor cells in patients with glioblastomas: A potential for targeted therapy? Int J Cancer 128: 826-838, 2011
43. Denysenko T, Gennero L, Roos MA, Melcarne A, Juenemann C, Faccani G, Morra I, Cavallo G, Reguzzi S, Pescarmona G, et al: Glioblastoma cancer stem cells: Heterogeneity, microenvironment and related therapeutic strategies. Cell Biochem Funct 28: 343-351, 2010.

44. Lee JH, Jung C, Javadian-Elyaderani P, Schweyer S, Schütte D, Shoukier M, Karimi-Busheri F, Weinfeld M, Rasouli-Nia A, Hengstler JG, et al: Pathways of proliferation and antiapoptosis driven in breast cancer stem cells by stem cell protein piwil2 Cancer Res 70: 4569-4579, 2010.

45. Honoki K, Fujii H, Kubo A, Kido A, Mori T, Tanaka Y and Tsujiuchi T: Possible involvement of stem-like populations with elevated ALDH1 in sarcomas for chemotherapeutic drug resistance. Oncol Rep 24: 501-505, 2010.

46. Masuda M, Wakasaki T, Suzui M, Toh S, Joe AK and Weinstein IB: Stat 3 orchestrates tumor development and progression: The Achilles' heel of head and neck cancers? Curr Cancer Drug Targets 10: 117-126, 2010.

47. Yao Z and Mishra L: Cancer stem cells and hepatocellular carcinoma. Cancer Biol Ther 8: 1691-1698, 2009.

48. Fang X, Cai Y, Liu J, Wang Z, Wu Q, Zhang Z, Yang CJ, Yuan L and Ouyang G: Twist 2 contributes to breast cancer progression by promoting an epithelial-mesenchymal transition and cancer stem-like cell self-renewal. Oncogene 30: 4707-4720, 2011

49. Hii CS, Anson DS, Costabile M, Mukaro V, Dunning K and Ferrante A: Characterization of the MEK5-ERK5 module in human neutrophils and its relationship to ERK1/ERK2 in the chemotactic response. J Biol Chem 279: 49825-49834, 2004.

50. Sato Y, Harada K, Kizawa K, Sanzen T, Furubo S, Yasoshima M, Ozaki S, Ishibashi M and Nakanuma Y: Activation of the MEK5/ ERK5 cascade is responsible for biliary dysgenesis in a rat model of Caroli's disease. Am J Pathol 166: 49-60, 2005. 